\title{
ARTICLE \\ Plasma miR-142 predicts major adverse cardiovascular events as an intermediate biomarker of dual antiplatelet therapy
}

Qian-jie Tang ${ }^{1,2,3}$, He-ping Lei ${ }^{1,2}$, Hong Wu ${ }^{4}$, Ji-yan Chen ${ }^{1,2}$, Chun-yu Deng ${ }^{1,2}$, Wang-sheng Sheng ${ }^{1}$, Yong-heng Fu ${ }^{1,2}$, Xiao-hong Li $^{1,2}$, Yu-bi Lin ${ }^{1,2}$, Ya-ling $\mathrm{Han}^{5}$ and Shi-long Zhong ${ }^{1,2}$

MicroRNAs (miRNAs) are widely expressed in organisms and are implicated in the regulation of most biological functions. The present study investigated the association of plasma miRNAs with the clinical outcomes of dual antiplatelet therapy in coronary artery disease (CAD) patients who underwent percutaneous coronary intervention (PCI). Plasma miRNA levels were screened using high-throughput Illumina sequencing to evaluate the antiplatelet efficacy of clopidogrel and aspirin. Six plasma miRNAs (miR-126, miR-130a, miR-27a, miR-106a, miR-21, and miR-142) were associated with clopidogrel-treated platelet aggregation. These miRNAs were validated in a prospective cohort of 1230 CAD patients using quantitative reverse transcription-polymerase chain reaction (qRT-PCR). High plasma miR-142 levels were associated with a high risk of major adverse cardiovascular events (MACE), with a hazard ratio (95\% confidence interval) of $1.83(1.30-2.59)$ at a false discovery rate of $<5 \%$. Multivariable Cox regression analysis revealed that diabetes mellitus, heart failure, calcium channel blocker application, and a high plasma miR-142 level were independent risk factors of MACE. The levels of the six plasma miRNAs were not significantly associated with bleeding events during the 3-year follow-up. In conclusion, plasma miR-142 is potential marker to predict MACE in CAD patients after PCI.

Keywords: plasma miRNAs; miR-142; antiplatelet therapy; MACEs; coronary artery disease

Acta Pharmacologica Sinica (2019) 40:208-215; https://doi.org/10.1038/s41401-018-0041-7

\section{INTRODUCTION}

Dual antiplatelet therapy using aspirin and clopidogrel is the standard of care for patients suffering from coronary artery disease (CAD) and undergoing percutaneous coronary intervention (PCI) [1]. However, the pharmacodynamic effects of clopidogrel vary substantially among individuals, and patients with reduced platelet inhibition are at an increased risk of major adverse cardiovascular events (MACEs) [2]. Some patients also experience drug-induced bleeding because of excessive platelet inhibition [3].

MicroRNAs (miRNAs), which are small noncoding RNA molecules approximately 22 nucleotides long, can play important regulatory roles in complex biological processes linked to multiple cardiovascular pathological conditions, including left ventricular hypertrophy, ischemic heart disease, heart failure, hypertension, and arrhythmias [4]. miRNAs stably exist in the blood, including the plasma, platelets, red blood cells, and nucleated blood cells, and they evade degradation by endogenous RNA polymerase [5]. miRNAs recognize their target mRNAs based on sequence complementarity and act at these sites to inhibit protein translation via mRNA degradation [6]. In clinical practice, miRNAs may be used as relevant biomarkers for diagnostic, prognostic, and monitoring purposes.
This study investigated the association of plasma miRNAs with the antiplatelet efficacy of clopidogrel and aspirin by evaluating the risk of MACE and the occurrence of bleeding events in CAD patients after $\mathrm{PCl}$.

\section{MATERIALS AND METHODS}

Study subjects and ethics statement

CAD patients were sequentially enrolled in Guangdong General Hospital from 2010 to 2014. CAD was diagnosed on the basis of angiographically documented coronary artery stenosis ( $\geq 50 \%$ luminal stenosis). All patients received dual antiplatelet therapy of clopidogrel and aspirin after $\mathrm{PCl}$ surgery. Patients were unrelated male and female Han Chinese between 40 and 80 years old. Demographic data, medical history, and medications were collected from the hospital information database.

The following exclusion criteria were used: advanced cancer, renal failure, hepatic failure, pregnancy, lactating, planning to become pregnant, any contraindication to aspirin or clopidogrel therapy, and preexisting bleeding disorders.

The study protocol was performed in accordance with the principles of the Declaration of Helsinki, and the Ethics Committee

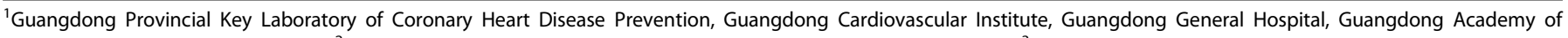

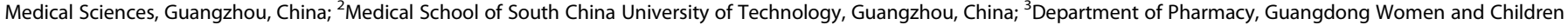

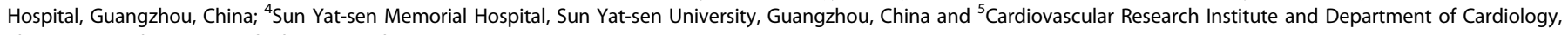
Shenyang Northern Hospital, Shenyang, China

Correspondence: Ya-ling Han (hanyaling@263.net) or Shi-long Zhong (zhongsl@hotmail.com)

These authors contributed equally: Qian-jie Tang, He-ping Lei, Hong Wu.

Received: 26 December 2017 Accepted: 6 April 2018

Published online: 11 June 2018 


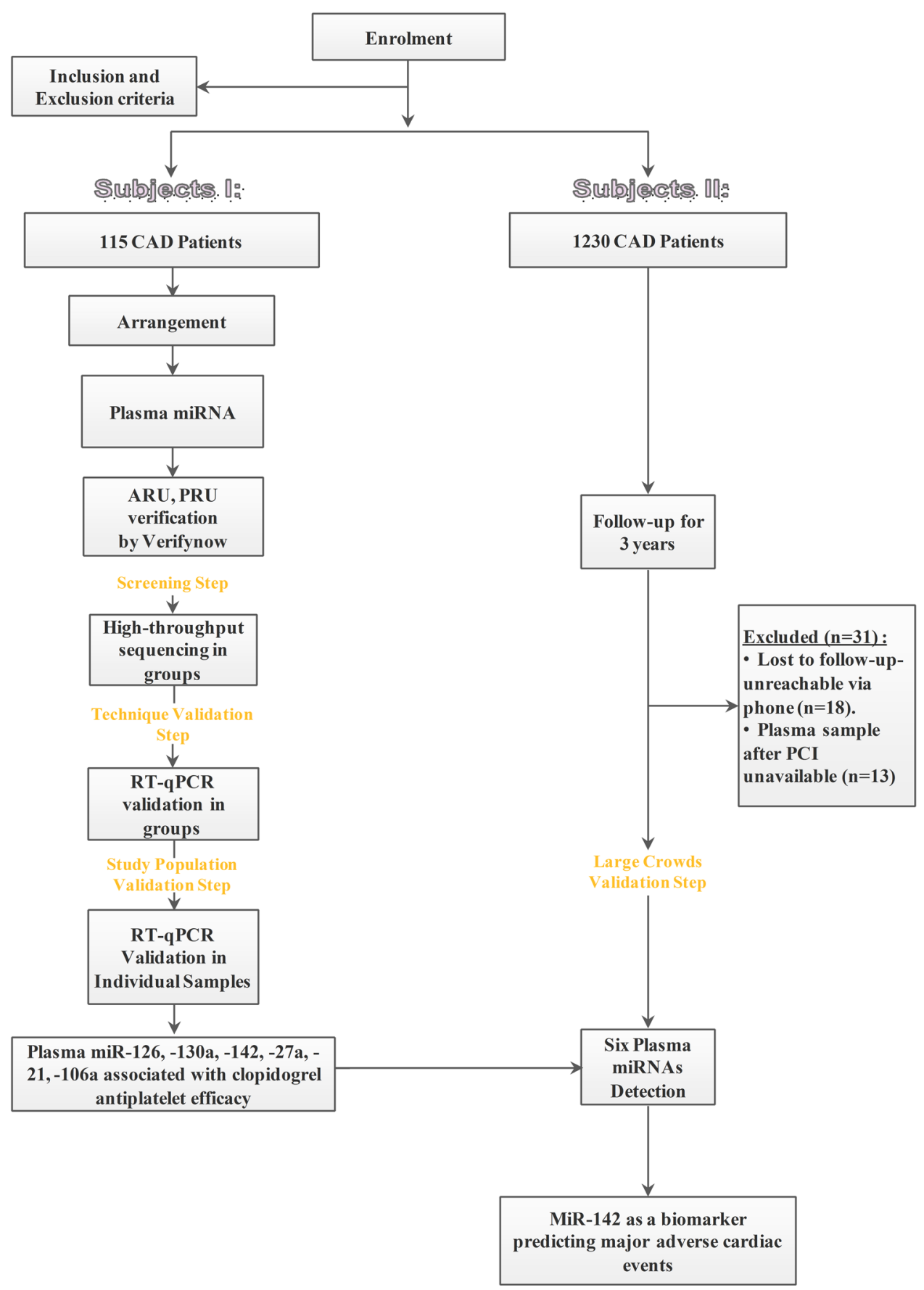

Fig. 1 Flowchart of the technological process. CAD, coronary artery disease; PCI, percutaneous coronary intervention; ARU, aspirin reaction unit; PRU, P2Y12 reaction unit

of Guangdong General Hospital approved the protocol. This study of 1230 CAD patients is registered in the Chinese Clinical Trial Registry (Registration number: ChiCTR-OCH-11001198). Each patient provided written informed consent to participate in the study.

Patient management and technological processes

This study involved two phases: (1) miRNA sequencing and (2) validation of the laboratory technique. A total of 115 CAD patients received $300 \mathrm{mg}$ of a loading dose of clopidogrel and $100 \mathrm{mg}$ of aspirin in the miRNA sequencing phase. Platelet aggregation rate units, including aspirin reaction unit (ARU) and $P 2 Y 12$ reaction unit (PRU), were detected at $4 \mathrm{~h}$ using the VerifyNow (Accumetrics Inc.,
San Diego, CA, USA) assay. Patients were divided into four subgroups according to low or high ARU or PRU. The plasma from each subgroup was pooled in equal volumes, and total RNA was extracted. Pooled plasma miRNA levels were measured using high-throughput Illumina sequencing. A technique validation step by quantitative reverse transcription polymerase chain reaction (qRT-PCR) was designed to validate the plasma miRNA levels in each subgroup, and the plasma miRNA level was verified individually in 115 CAD patients using qRT-PCR. The screened miRNAs were subsequently validated using qRT-PCR in a prospective cohort of 1230 CAD patients who were followed up for 3 years.

Figure 1 shows the flowchart of this procedure. 
Study endpoints and follow-up

Follow-up information was collected on the basis of inpatient and outpatient hospital visits and telephone contacts with patients or their families. The primary efficacy and safety outcomes were used as study endpoints. The primary endpoints were defined as MACEs, including cardiovascular death, myocardial infarction, or stent thrombosis, during a 3-year follow-up period. The records of patient's clinical endpoints were registered 6, 12, 24, and 36 months after $\mathrm{PCl}$. Clinical safety outcomes were major and minor bleeding events defined according to Serebruany et al. [7].

\section{Antiplatelet effect analysis}

The antiplatelet effectiveness of clopidogrel and aspirin was measured using the VerifyNow assay as described by the manufacturer. This instrument measures light transmittance as aggregation occurs, and data are converted into ARU or PRU. High on-treatment platelet reactivity (HTPR) is associated with worse outcomes, and HTPR was defined as PRUs > 208 for clopidogrel and ARUs $>550$ for aspirin [8].

\section{miRNA extraction}

Blood samples were collected and anticoagulated using ethylenediamine tetraacetic acid dipotassium salt. Blood samples were centrifuged at 3000 revolutions per minute for $10 \mathrm{~min}$ to remove the blood cells, and the supernatant was transferred to microcentrifuge tubes, followed by a second centrifugation at 12,000 revolutions per minute for $10 \mathrm{~min}$. Plasma was harvested, aliquoted and stored at $-80^{\circ} \mathrm{C}$ until analysis. Total RNA, which contained miRNA, was isolated from $200 \mu \mathrm{L}$ of the plasma using the mirVana PARIS kit (Ambion, Foster City, CA, USA) according to the manufacturer's instructions. The concentrations of all RNA samples were quantified using a NanoDrop 2000 (Thermo Fisher Scientific, Waltham, MA, USA).

Plasma miRNAs discovered by high-throughput sequencing and validated by qRT-PCR

Fifty-six of the 115 enrolled patients (48.7\%) were clopidogrelresistant, and 51 patients (44.35\%) were aspirin-resistant. The patients were divided into 4 subgroups based on low and high ARU and PRU: 29 patients (25.22\%) in the LowARU LowPRU group (ARU < 550, PRU < 208), 35 patients $(30.43 \%)$ in the LowARU HighPRU group (ARU < 550, PRU $>208$ ), 30 patients $(26.09 \%$ ) in the HighARU LowPRU group (ARU $>550$, PRU $<208$ ), and 21 patients (18.26\%) in the HighARU HighPRU group (ARU > 550, PRU $>208)$. Equal volumes of plasma $(20 \mu \mathrm{L})$ from 20 individuals were mixed in the same subgroup. We extracted the total RNA from each subgroup for high-throughput sequencing.

The BGI Company (Shenzhen, China) performed the highthroughput sequencing and analyzed the plasma miRNA levels from the mixed pool. This sequencing method was used to screen the plasma miRNAs that exhibited differences in expression. Two biological replicates and two technical replicates were performed.

RNA sequencing is a powerful technique for discovery-phase research, but qRT-PCR is superior to many miRNA quantification methods $[9,10]$. Plasma concentrations of miR-126, miR-130a, miR-27a, miR-106a, miR-21, and miR-142 were determined by qRTPCR. Total RNA $(2 \mu \mathrm{L})$ was reverse-transcribed using the PrimeScript RT Reagent Kit (Takara, Japan) in accordance with the manufacturer's protocol. The qRT-PCR results were analyzed using Bulge-Loop $^{\text {TM }}$ miRNA primers (Ribobio Co, Guangzhou, China) and iTaq Universal SYBR Green Supermix (Bio-Rad, Hercules, CA, USA), in accordance with the manufacturer's protocol, in a Bio-Rad CFX Connect Real-Time PCR Detection System (Bio-Rad, Hercules, CA, USA). Cel-miR-39 was used as an endogenous control. The relative expression levels of miRNAs were normalized to cel-miR-39 and calculated using the equation $2^{-\Delta \mathrm{Ct}}$, where $\Delta \mathrm{Ct}=$ mean $\mathrm{Ct}_{\text {miRnA }}{ }^{-}$mean $\mathrm{Ct}_{\text {cel-miR-39. }} \Delta \Delta \mathrm{Ct}$ was calculated by subtracting the mean $\Delta \mathrm{Ct}$ of the control samples from the $\Delta \mathrm{Ct}$ of tested samples. Fold- changes in miRNA were calculated by the equation $2^{-\Delta \Delta \mathrm{Ct}}$. All measurements were performed in duplicate, and the mean $\mathrm{Ct}$ values were calculated. Replicates/wells with $\mathrm{Ct}>35$ were excluded.

\section{Statistical analysis}

Demographic and clinical characteristics are summarized using counts (percentages) for categorical variables and the means \pm standard deviation (SD) for continuous variables. The normality of the distribution of continuous variables was checked using the Shapiro-Wilk normality test. Logarithmic transformation was performed prior to analysis if the ranges of the dependent variables were skewed. Differences in baseline characteristics between patients who were sensitive and resistant to aspirin or clopidogrel were compared using logistic regression analysis. Spearman correlation analysis was calculated to describe the correlation between PRU and plasma miRNA expression levels. The two-tailed Mann-Whitney test was used to compare plasma miRNA expression levels between patients with and without clopidogrel resistance. A univariate logistic regression analysis was performed to evaluate the independent effects of baseline demographic and clinical characteristics and plasma miRNA levels on the risk of bleeding and to calculate odds ratios (ORs) and 95\% confidence intervals (Cls). Cox regression analyses were used to evaluate the effects of clinical characteristics on MACE. Variables with $P<0.10$ were entered into the multivariable Cox regression model, and only variables with $P<0.10$ were retained in the model. Variables included age, gender (coded as $0 / 1$ ), diabetes mellitus, hypertension, heart failure (coded as $0 / 1$ ) and use of medications (coded as $0 / 1$ ). A false discovery rate (FDR) control was used to correct for multiple comparisons using the SAS PROC MULTTEST with the FDR option. $P$-values $<0.05$ were considered statistically significant, and the FDR was controlled to the 0.05 level. Data analyses were performed using SAS 9.1 (SAS Institute, Cary, NC, USA).

\section{RESULTS}

Clinical trial cohort for screening plasma miRNAs related to dual antiplatelet efficacy

A total of 115 CAD patients who were enrolled in Guangdong General Hospital from October 2013 to January 2014 received a $300-\mathrm{mg}$ loading dose of clopidogrel and $100 \mathrm{mg}$ of aspirin. All patients were unrelated male and female Han Chinese patients between 54 and 75 years old.

Supplementary Table S1 and Table S2 show the differences in baseline characteristics between patients sensitive and resistant to aspirin and clopidogrel. Higher AST and CKMB levels were significantly associated with lower ARUs, with an OR $(95 \% \mathrm{Cl})$ of $0.97(0.94-1.00)(P=0.0416)$ and OR $(95 \% \mathrm{Cl})$ of $0.91(0.83-0.99)(P$ $=0.0301)$, respectively, which indicates that patients with higher AST and CKMB were more sensitive to aspirin (Table S1). The effects of statin application were similarly associated with aspirin sensitivity, with an OR (95\%) of $0.12(0.00-0.63) \quad(P=0.0284)$. Higher LDLC and CHOL levels were significantly associated with higher PRUs, with ORs $(95 \% \mathrm{Cl})$ of 1.67 (1.04-2.67) and 1.47 (1.02-2.13) (all $P<0.05$ ), respectively (Table S2). These results demonstrated that LDLC and $\mathrm{CHOL}$ were risk factors for clopidogrel resistance.

Plasma miRNA discovered by high-throughput sequencing and validated by qRT-PCR

The differential expression of known miRNAs in the four groups (LowARU LowPRU, LowARU HighPRU, HighARU LowPRU and HighARU HighPRU groups) was compared to identify differentially expressed miRNAs. We anticipated that the expression of miRNAs in the LowARU HighPRU and HighARU HighPRU groups would be higher than the corresponding control groups. The Illumina 
sequencing revealed that 17 miRNAs complied with our expected trend: miR-27a-3p, miR-17-3p, miR-17-5p, miR-21-3p, miR-21-5p, miR-28-5p, miR-32-5p, miR-101-3p, miR-106a-5p, miR-126-5p, miR130a-3p, miR-142-5p, miR-181c-3p, miR-184, miR-192-5p, miR-342$3 p$, and Let-7i-5p. These 17 miRNAs were validated by qRT-PCR. miR-32-5p, miR-28-5p, miR-21-3p, miR-181c-3p, and miR-17-3p were undetectable under the same experimental conditions because of undesirable amplification and melt curves. qRT-PCR of the remaining 12 miRNAs demonstrated that half of the miRNAs (miR-21-5p, miR-27a-3p, miR-106a-5p, miR-126-5p, miR-130a-3p, and miR-142-5p) were upregulated in the same pools, which was consistent with the Illumina sequencing results. Six other miRNAs (miR-17-5p, let-7i-5p, miR-101-3p, miR-184, miR-192-5p, and miR$342-3 p)$ showed inconsistent results with the Illumina sequencing and were excluded from further analysis.

Table S3A and Table S3B show the patient characteristics and their associations with the levels of 6 plasma miRNAs. Females were associated with a lower plasma level of miR-126-5p with an estimate of $-0.2816(P=0.0246)$ in univariate analysis. Comorbidity of diabetes was associated with higher plasma levels of miR-126-5p (Estimate $=0.2773, P=0.0133$ ) and miR-130a-3p (Estimate $=0.2967, P=0.0257$ ). A higher level of apolipoprotein A (apoA) was associated with a lower plasma level of miR-21-5p (Estimate $=-0.5878, P=0.0063$ ), and a higher level of creatine kinase (CK) was associated with a lower plasma level of miR-126$5 p$ (Estimate $=-0.0004, P=0.0335$ ). A higher level of highdensity lipoprotein cholesterol (HDLC) was associated with higher plasma levels of miR-126-5p (Estimate $=0.4498, P=$ 0.0262 ) and miR-130a-3p (Estimate $=0.6687, P=0.0053$ ). The effect of the application of proton pump inhibitors (PPIs) was significantly associated with higher plasma levels of miR-27a-3p, miR-126-5p, miR-130a-3p and miR-142-5p ( $P=0.0005,0.0440$, $0.0109,0.0444$, respectively).

Multivariate analysis demonstrated that $\mathrm{CK}, \mathrm{HDLC}$, diabetes, and PPIs application were independent factors of the variability of plasma miR-126-5p levels $(P=0.0147,0.0418,0.0046$, and 0.0263 , respectively). HDLC, diabetes and application of PPIs were independent factors that influenced the variability in plasma miR-130a-3p levels ( $P=0.0059,0.0479$, and 0.0122 , respectively).

Plasma miRNAs were associated with clopidogrel-treated platelet aggregation

Figure 2 shows the correlation between PRU and the expression levels of the six validated miRNAs in all 115 CAD patients. miR-126, miR-142, miR-130a, miR-27a, and miR-21 exhibited levels that were significantly associated with PRU $(P<0.05)$. Further analysis indicated that the plasma levels of miR-126, miR-130a, miR-142, miR-27a, and miR-106a were high in individual patients resistant to clopidogrel treatment $(P<0.05$; Fig. 3). The expression levels of miR-27a, miR-126, and miR-130a were significantly increased in patients resistant to clopidogrel and sensitive to aspirin $(P<0.05$; Fig. 4). The expression level of miR-21 was significantly decreased in patients resistant to clopidogrel $(P<0.05)$. However, among patients who were resistant to aspirin, the miR-126, miR-142, miR130a, miR-27a, and miR-21 levels were significantly higher in patients resistant to clopidogrel than in patients sensitive to clopidogrel $(P<0.05$; Fig. 5).

Predicting the value of plasma miRNA on MACE in CAD patients A total of 1230 CAD patients undergoing $\mathrm{PCl}$ were sequentially enrolled in Guangdong General Hospital during March 2010 to June 2014. All CAD patients were between 53 and 75 years old and were unrelated Han Chinese. A total of 31 patients who were lost to follow-up (e.g., unreachable via phone; $n=18$ ) or whose plasma samples after $\mathrm{PCl}$ were unavailable $(n=13)$ were excluded. The remaining 1199 patients were investigated. Table S4 shows the baseline clinical characteristics and their effects on MACE in 1199 patients after PCl. Their mean age was 63.75 years, and approximately $80.23 \%$ were males. The use of CCBs (hazard ratio $[\mathrm{HR}]=2.48,95 \%$ confidence interval $[\mathrm{Cl}]=1.60-3.82, P<0.0001$ ) and PPIs ( $\mathrm{HR}=1.87,95 \% \mathrm{Cl}=1.17-2.99, P=0.0091)$ was associated with a significantly higher risk of MACE in patients after $\mathrm{PCl}$.

All six miRNAs were present at quantifiable levels in the plasma samples of the prospective cohort. A high miR-142 level was
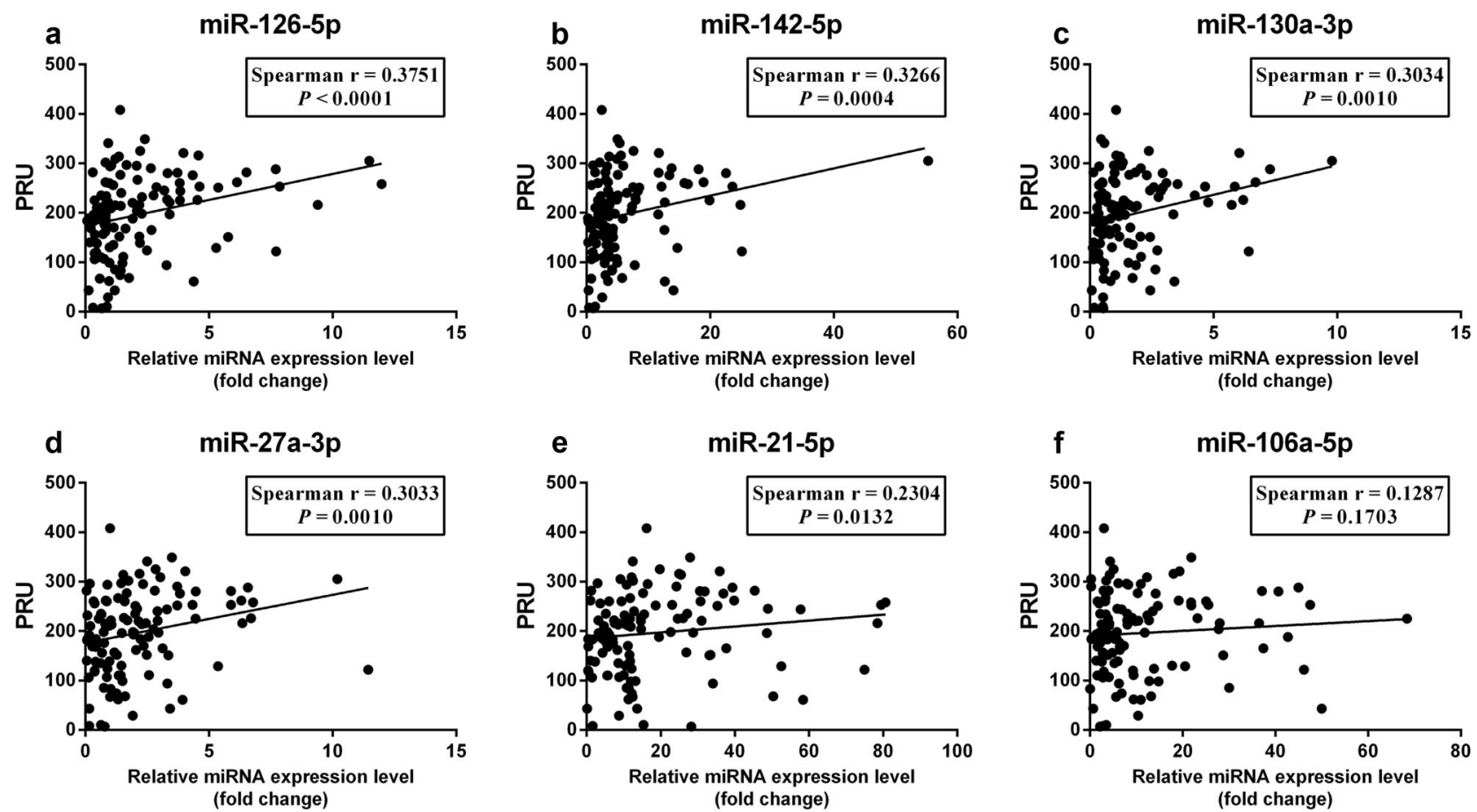

Fig. 2 Correlations between PRU and plasma miR-126 (a), miR-142 (b), miR-130a (c), miR-27a (d), miR-21 (e), and miR-106a (f) expression levels in 115 CAD patients 

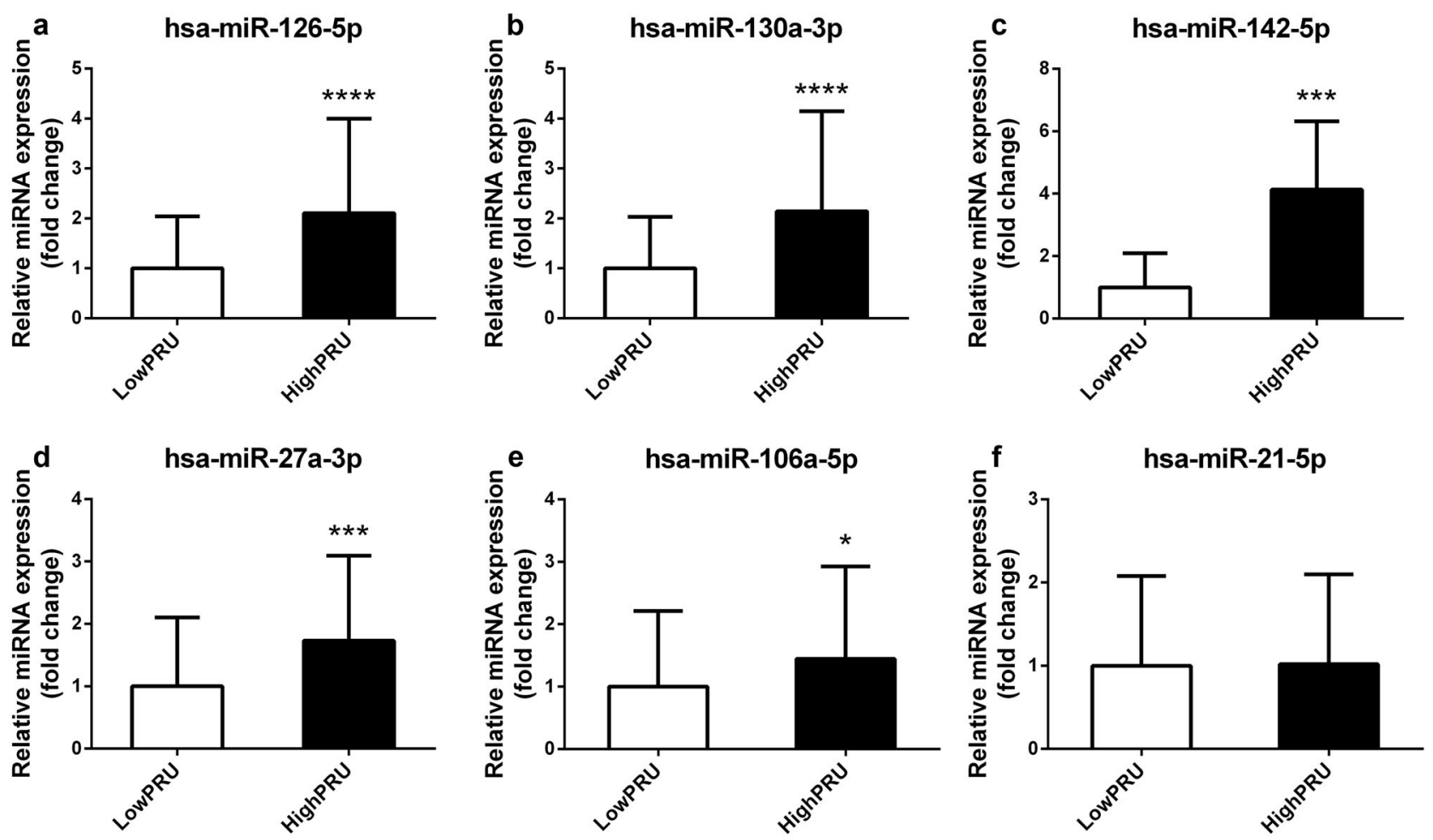

Fig. 3 Plasma miR-126 (a), miR-130a (b), miR-142 (c), miR-27a (d), miR-106a (e), and miR-21 (f) levels in individual patients sensitive or resistant to clopidogrel treatment. ${ }^{* * *} P<0.0001,{ }^{* * *} P<0.001,{ }^{*} P<0.05$ versus low PRU
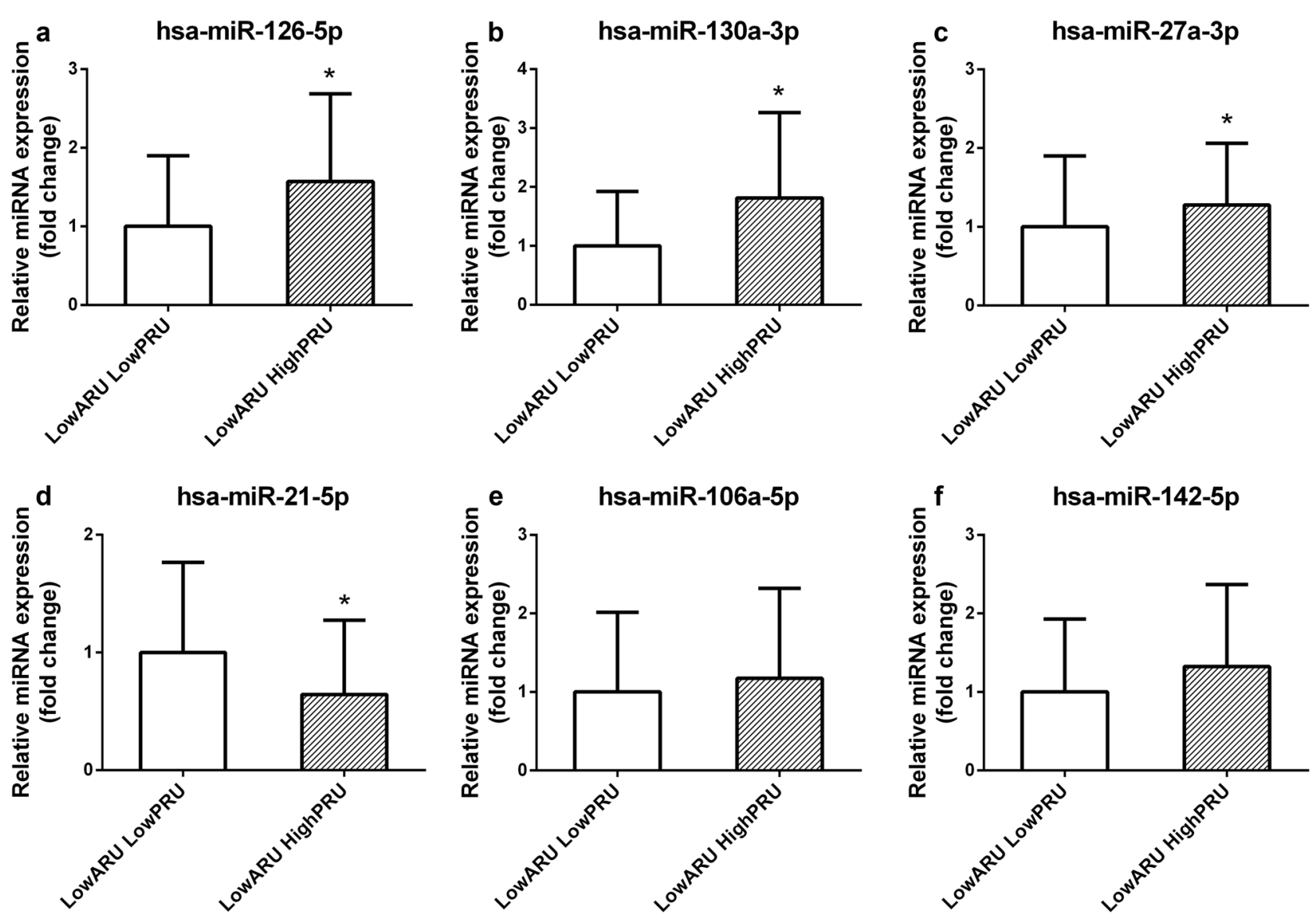

Fig. 4 Patients were grouped by sensitivity to aspirin to determine the expression levels of plasma miR-126 (a), miR-130a (b), miR-27a (c), miR21 (d), miR-106a (e), and miR-142 (f) between patients sensitive and resistant to clopidogrel. ${ }^{*} P<0.05$ versus low ARU and low PRU 

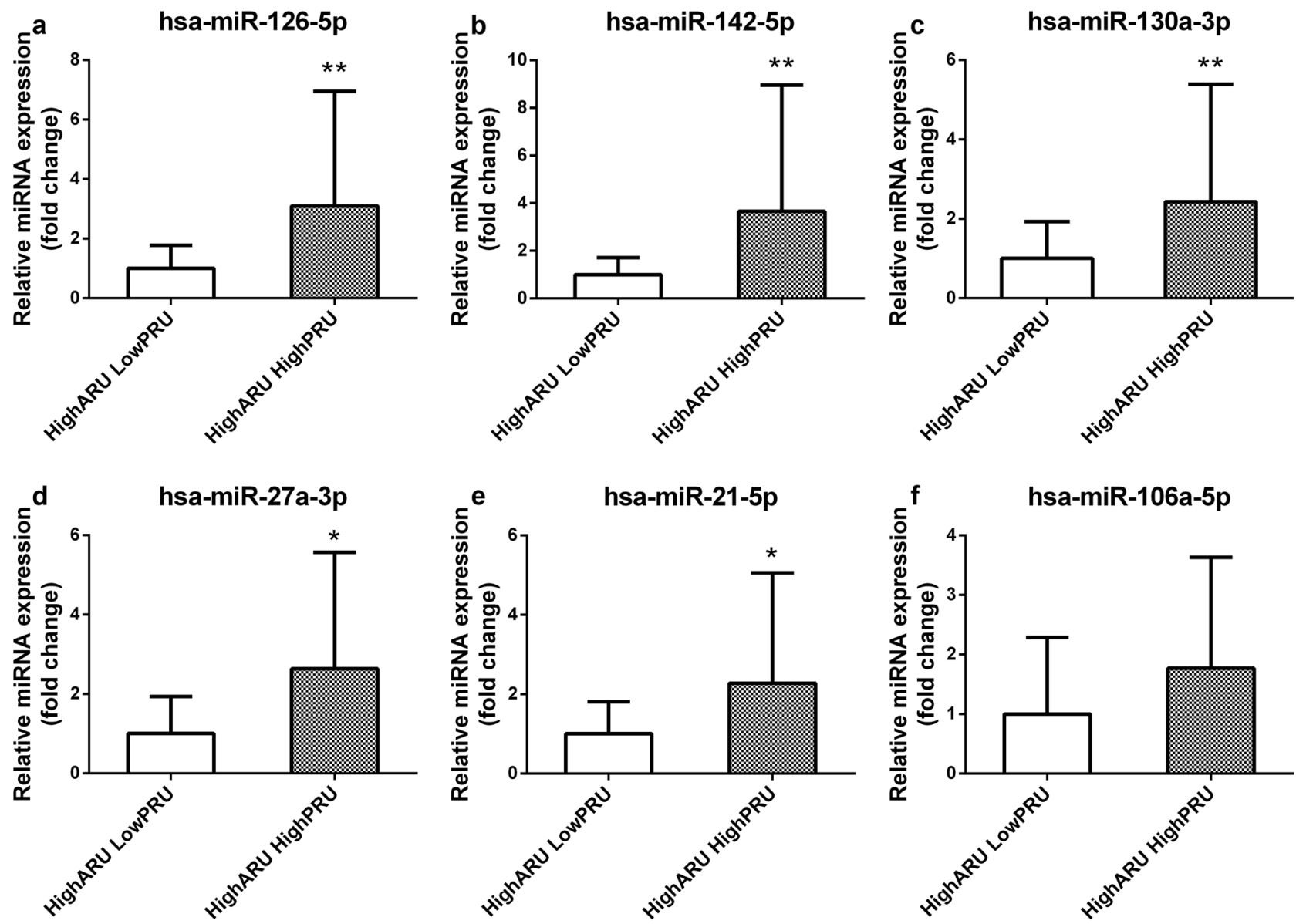

Fig. 5 Patients were grouped by resistance to aspirin to determine the expression levels of plasma miR-126 (a), miR-142 (b), miR-130a (c), miR27 a (d), miR-21 (e), and miR-106a (f) between patients sensitive and resistant to clopidogrel. ${ }^{* *} P<0.01,{ }^{*} P<0.05$ versus high ARU and low PRU

Table 1. Expression levels of six miRNAs in the plasma of the prospective cohort and their effects on MACE

\begin{tabular}{lllll}
\hline Characteristics & $\begin{array}{l}\text { Fold change } \\
\text { (Mean } \pm \text { SD) }\end{array}$ & HR $(95 \% \mathrm{Cl})$ & $P$ & FDR \\
\hline hsa-miR-21-5p & $0.38 \pm 0.75$ & $1.08(0.84-1.40)$ & 0.5400 & 0.5538 \\
hsa-miR-27a-3p & $0.10 \pm 0.19$ & $1.35(0.50-3.63)$ & 0.5538 & 0.5538 \\
hsa-miR-106a-5p & $0.25 \pm 0.58$ & $1.11(0.83-1.49)$ & 0.4779 & 0.5538 \\
hsa-miR-126-5p & $0.04 \pm 0.04$ & $5.01(1.12-22.47)$ & 0.0353 & 0.1059 \\
hsa-miR-130a-3p & $0.07 \pm 0.18$ & $1.47(0.59-3.64)$ & 0.4084 & 0.5538 \\
hsa-miR-142-5p & $0.15 \pm 0.35$ & $1.83(1.30-2.59)$ & 0.0006 & 0.0036 \\
\hline FDR false discovery rate & & & \\
\hline
\end{tabular}

associated with a high risk of MACE, with an $\mathrm{HR}(95 \% \mathrm{Cl})$ of 1.83 (1.30-2.59) at a FDR of $<5 \%$ (Table 1). Multivariable Cox regression analysis revealed that a high plasma miR-142 level, diabetes mellitus, heart failure, and the use of CCBs were independent risk factors for MACE, with HRs $(95 \% \mathrm{Cl})$ of 1.92 (1.37-2.69), 1.73 (1.12-2.67), 3.13 (1.91-5.11), and 2.07 (1.34-3.18), respectively (Table 2).

The value of plasma miRNA in predicting bleeding events in CAD patients

The primary safety endpoint of bleeding during the follow-up period occurred in 249 patients (20.8\%), including 122 patients with petechia or ecchymosis events, 68 patients with melaena or gastrointestinal bleeding events, 55 patients with unusual events of bleeding from the mouth or gums, 2 patients with epistaxis events, and 2 patients with hematuria events.

Table S5 shows the baseline characteristics of the prospective cohort according to bleeding status. Univariate analysis revealed that LDLC (OR $=1.17 ; 95 \% \mathrm{Cl}=1.01-1.34)$ and the use of PPIs (OR $=1.58 ; 95 \% \mathrm{Cl}=1.19-2.12$ ) were associated with a high risk of bleeding events. However, the expression level of plasma miR-21, miR-27, miR-106a, miR-126, miR-130a, or miR-142 did not significantly affect the bleeding events in CAD patients $(P>0.05)$.

\section{DISCUSSION}

The results of this study showed that high plasma miR-142 levels were associated with a significantly high risk of MACE in dual antiplatelet-treated patients undergoing $\mathrm{PCl}$. However, no significant association was observed between the levels of six plasma miRNAs and the bleeding events during the 3-year follow-up.

Patients in the initial screening phase were divided into four groups according to low or high ARU or PRU, and an equal volume of plasma from each group was pooled. The pooling method has the advantage that it allows the detection of miRNAs that are expressed in most of the subjects in each group. This method of pooling plasma samples is a widely used technique that is reliable for profiling, and it reduces sample variation and enriches miRNAs that are most likely to change between groups [11-14].

Accumulating evidence suggests that miR-142-5p is hematopoietic-specific in the circulation [15-17]. It is a key regulator in macrophage apoptosis, cardiac hypertrophy, atherosclerosis, and heart failure [18-20]. Platelet extracellular vesicles 
Table 2. Effect of plasma miRNA on MACE in the prospective cohort by applying a multivariate Cox regression analysis

\begin{tabular}{lllrrr}
\hline Characteristics & $B$ & Standard error & Wald $x^{2}$ & $\mathrm{HR}(95 \% \mathrm{Cl})$ \\
\hline hsa-miR-142-5p & 0.65054 & 0.17253 & 14.2174 & $1.92(1.37-2.69)$ & 0.0002 \\
Calcium channel blockers & 0.72619 & 0.22024 & 10.8717 & $2.07(1.34-3.18)$ \\
Diabetes mellitus & 0.55021 & 0.22108 & 6.1941 & $1.73(1.12-2.67)$ \\
Heart failure & 1.13972 & 0.25077 & 20.6568 & $3.13(1.91-5.11)$ & 0.0010 \\
\hline
\end{tabular}

exhibit upregulated miR-142-5p, which is involved in vascular and metabolic disease [21]. miR-142 is suppressed in a hypertrophic heart model and human cardiomyopathic hearts [22]. miR-142-5p was upregulated in heart failure patients undergoing implantation of a left ventricular assisting device. The identified miRNAs may contribute to the molecular regulation of reverse remodeling and heart recovery mechanisms [20]. The inhibition of miR-142-5p attenuated OGD/R-induced cell injury and oxidative stress, and overexpression of miR-142-5p aggravated these conditions [23]. Overexpression of miR-142-5p during hypoxia produced extensive cell injury and apoptosis, and suppression of miR-142-5p significantly promoted cell viability and attenuated cell apoptosis [24]. Xu et al. [18] demonstrated that the upregulation of miR-142$5 p$ expression regulated apoptosis in human macrophages via the targeting of TGF- $\beta 2$ in the progression of atherosclerosis. Sharma et al. [19] reported that the forced expression of miR-142 during hypertrophic growth produced extensive apoptosis and cardiac dysfunction. Barsanti et al. [20] found that the expression levels of identified miRNAs, including miR-142-5p, were correlated with offpump cardiac index values and may contribute to the molecular regulation of reverse remodeling and heart recovery mechanisms. Molecular function analyses revealed that the B-cell translocation gene may be a direct target of miR-142-5p, which regulates vascular cell proliferation [25]. We hypothesized significant associations among miR-142, platelet reactivity and the risk of cardiovascular events based on the role of miR-142 in cardiovascular diseases. The present study demonstrated that high plasma miR-142 levels were associated with a high risk of MACE. Further cytological experiments are necessary to elucidate the molecular mechanisms of miR-142 in the regulation of platelet function.

Platelets contain and release miRNAs and are a major source of miRNAs in plasma. Previous studies demonstrated that platelet activation triggered the release of miRNAs into circulation, and antiplatelet therapy reduced plasma levels of platelet-related miRNAs [26, 27]. Shi et al. [28] suggested that a blunted response to the P2Y12 antagonist, that was, HTPR, was a strong predictor of MACEs in patients with coronary heart disease receiving antiplatelet therapy. Our results are consistent with these data. In this study, miR-142-5p was upregulated in plasma samples of patients with high PRU, which suggests an increased release of miR-142 as a resistance response to antiplatelet treatment. Our data also demonstrated that plasma miR-142 was a potential biomarker to evaluate the antiplatelet efficacy of clopidogrel and aspirin and predict MACE as the clinical endpoint.

The use of CCBs and PPIs was associated with a significantly high risk of MACE in patients after PCI. Previous studies reported that the concomitant use of CCBs and PPIs inhibited the antiplatelet activity of clopidogrel because of the inhibition of CYP3A4 and CYP2C19 isoenzymes, respectively [29-31]. More studies are needed to confirm our findings. However, physicians should exercise appropriate caution when prescribing CCBs or PPIs for patients who have recently undergone $\mathrm{PCl}$ and receive clopidogrel.

There were some limitations in this study. First, our findings should be cautiously interpreted because all of the subjects were from the same ethnic background and geographical region. Second, there was a lack of detection of the association of plasma
miR-142 levels with clopidogrel efficiency in the 1230 patients. Third, we only discovered the phenomenon of plasma miRNA via evaluation of the antiplatelet efficacy of clopidogrel, and the precise mechanisms remain unknown.

In conclusion, plasma miR-142 was identified as an intermediate biomarker to evaluate the antiplatelet efficacy of clopidogrel and predict MACE in CAD patients after $\mathrm{PCl}$. Our results provide a new basis for the development of appropriate biological indicators corresponding to the efficacy of antiplatelet treatment in vivo.

\section{ACKNOWLEDGEMENTS}

This work was supported by the National Key R\&D program (No 2017 YFC0909301 and 2016YFC0905003), National Natural Science Foundation of China (No 81673514, 81373486, 81202602, and 81470440), Science and Technology Development Projects of Guangdong Province, China (No 2016B090918114), and Science and Technology Development Projects of Guangzhou, Guangdong, China (201510010236 and 201604020096).

\section{AUTHORS CONTRIBUTIONS}

$\mathrm{SZ}, \mathrm{YH}, \mathrm{QT}, \mathrm{HL}$, and $\mathrm{HW}$ designed the research. $\mathrm{QT}, \mathrm{HL}$, and $\mathrm{HW}$ performed the experiments and wrote the article. SZ, YH, and QT analyzed the data. Other colleagues helped collect the sample and clinical data. All authors have read and approved the final manuscript.

\section{ADDITIONAL INFORMATION}

The online version of this article (https://doi.org/10.1038/s41401-018-0041-7) contains supplementary material, which is available to authorized users.

Competing interests: The authors declare no competing interests

\section{REFERENCES}

1. Beitelshees AL, Voora D, Lewis JP. Personalized antiplatelet and anticoagulation therapy: applications and significance of pharmacogenomics. Pharmgenomics Pers Med. 2015;8:43-61.

2. Bonello L, Tantry US, Marcucci R, Blindt R, Angiolillo DJ, Becker R, et al. Consensus and future directions on the definition of high on-treatment platelet reactivity to adenosine diphosphate. J Am Coll Cardiol. 2010;56:919-33.

3. Yusuf S, Zhao F, Mehta SR, Chrolavicius S, Tognoni G, Fox KK. Effects of clopidogrel in addition to aspirin in patients with acute coronary syndromes without ST-segment elevation. N Engl J Med. 2001;345:494-502.

4. Romaine SP, Tomaszewski M, Condorelli G, Samani NJ. MicroRNAs in cardiovascular disease: an introduction for clinicians. Heart. 2015;101:921-8.

5. Berezikov E, Cuppen E, Plasterk RH. Approaches to microRNA discovery. Nat Genet. 2006;38:S2-7.

6. Shenouda SK, Alahari SK. MicroRNA function in cancer: oncogene or a tumor suppressor? Cancer Metastas. 2009;28:369-78.

7. Serebruany VL, Atar D. Assessment of bleeding events in clinical trials-proposal of a new classification. Am J Cardiol. 2007;99:288-90.

8. Davila CD, Vargas F, Huang KH, Monaco T, Dimou A, Rangaswami J, et al. Dipstick proteinuria is an independent predictor of high on treatment platelet reactivity in patients on clopidogrel, but not aspirin, admitted for major adverse cardiovascular events. Platelets. 2015;26:651-6.

9. Pritchard $\mathrm{CC}$, Cheng $\mathrm{HH}$, Tewari M. MicroRNA profiling: approaches and considerations. Nat Rev Genet. 2012;13:358-69.

10. Kang K, Peng X, Luo J, Gou D. Identification of circulating miRNA biomarkers based on global quantitative real-time PCR profiling. J Anim Sci Biotechnol. 2012;3:4. 
11. Oka S, Furukawa H, Shimada K, Hashimoto A, Komiya A, Fukui N, et al. Plasma miRNA expression profiles in rheumatoid arthritis associated interstitial lung disease. BMC Musculoskelet Disord. 2017;18:21.

12. Wang R, Hong J, Cao Y, Shi J, Gu W, Ning G, et al. Elevated circulating microRNA122 is associated with obesity and insulin resistance in young adults. Eur J Endocrinol. 2015;172:291-300.

13. Chen Q, Ge X, Zhang Y, Xia H, Yuan D, Tang Q, et al. Plasma miR-122 and miR-192 as potential novel biomarkers for the early detection of distant metastasis of gastric cancer. Oncol Rep. 2014;31:1863-70.

14. Liu T, Zhong S, Rao F, Xue Y, Qi Z, Wu S. Catheter ablation restores decreased plasma miR-409-3p and miR-432 in atrial fibrillation patients. Europace. 2016;18:92-9.

15. Chen CZ, Li L, Lodish HF, Bartel DP. MicroRNAs modulate hematopoietic lineage differentiation. Science. 2004;303:83-6.

16. Landgraf P, Rusu M, Sheridan R, Sewer A, lovino N, Aravin A, et al. A mammalian microRNA expression atlas based on small RNA library sequencing. Cell. 2007;129:1401-14.

17. Merkerova M, Belickova $M$, Bruchova $H$. Differential expression of microRNAs in hematopoietic cell lineages. Eur J Haematol. 2008;81:304-10.

18. Xu R, Bi C, Song J, Wang L, Ge C, Liu X, et al. Upregulation of miR-142-5p in atherosclerotic plaques and regulation of oxidized low-density lipoproteininduced apoptosis in macrophages. Mol Med Rep. 2015;11:3229-34.

19. Sharma S, Liu J, Wei J, Yuan H, Zhang T, Bishopric NH. Repression of miR-142 by p300 and MAPK is required for survival signalling via gp130 during adaptive hypertrophy. EMBO Mol Med. 2012;4:617-32.

20. Barsanti C, Trivella MG, D'Aurizio R, El Baroudi M, Baumgart M, Groth $M$, et al Differential regulation of microRNAs in end-stage failing hearts is associated with left ventricular assist device unloading. Biomed Res Int. 2015;2015:592512.

21. Pienimaeki-Roemer A, Konovalova T, Musri MM, Sigruener A, Boettcher A, Meister $\mathrm{G}$, et al. Schmitz G. Transcriptomic profiling of platelet senescence and platelet extracellular vesicles. Transfusion. 2017;57:144-56.

22. Jiao $M$, You HZ, Yang $X Y$, Yuan $H$, Li YL, Liu WX, et al. Circulating microRNA signature for the diagnosis of childhood dilated cardiomyopathy. Sci Rep. 2018;8:724
23. Wang N, Zhang L, Lu Y, Zhang M, Zhang Z, Wang K, et al. Down-regulation of microRNA-142-5p attenuates oxygen-glucose deprivation and reoxygenationinduced neuron injury through up-regulating Nrf2/ARE signaling pathway. Biomed Pharmacother. 2017;89:1187-95.

24. Zhan L, Lei S, Li W, Zhang Y, Wang H, Shi Y, et al. Suppression of microRNA-142$5 p$ attenuates hypoxia-induced apoptosis through targeting SIRT7. Biomed Pharmacother. 2017;94:394-401.

25. Kee HJ, Park S, Kwon JS, Choe N, Ahn Y, Kook H, et al. B cell translocation gene, a direct target of miR-142-5p, inhibits vascular smooth muscle cell proliferation by down-regulating cell cycle progression. FEBS Lett. 2013;587:2385-92.

26. Kaudewitz D, Skroblin P, Bender LH, Barwari T, Willeit $P$, Pechlaner $R$, et al Association of MicroRNAs and YRNAs with platelet function. Circ Res. 2016;118:420-32.

27. Willeit P, Zampetaki A, Dudek K, Kaudewitz D, King A, Kirkby NS, et al. Circulating microRNAs as novel biomarkers for platelet activation. Circ Res. 2013;112:595-600.

28. Shi R, Zhou X, Ji WJ, Zhang YY, Ma YQ, Zhang JQ, et al. The emerging role of miR223 in platelet reactivity: implications in antiplatelet therapy. Biomed Res Int. 2015;2015:981841.

29. Gremmel T, Steiner S, Seidinger D, Koppensteiner R, Panzer S, Kopp CW. Calciumchannel blockers decrease clopidogrel-mediated platelet inhibition. Heart. 2010;96:186-9.

30. Ojeifo O, Wiviott SD, Antman EM, Murphy SA, Udell JA, Bates ER, et al. Concomitant administration of clopidogrel with statins or calcium-channel blockers: insights from the TRITON-TIMI 38 (trial to assess improvement in therapeutic outcomes by optimizing platelet inhibition with prasugrel-thrombolysis in myocardial infarction 38). JACC Cardiovasc Interv. 2013;6:1275-81.

31. Goodman SG, Clare R, Fau-Pieper KS, Pieper Ks, Fau-Nicolau JC, Nicolau Jc, et al. Association of proton pump inhibitor use on cardiovascular outcomes with clopidogrel and ticagrelor: insights from the platelet inhibition and patient outcomes trial. Circulation. 2012;125:978-86. 